\title{
Bounded Analytic Functions in the Dirichlet Space
}

\author{
Stefan Richter ${ }^{1}$ and Allen Shields ${ }^{2}$ \\ ${ }^{1}$ Department of Mathematics, University of Virginia, Charlottesville, VA 22903-3199, USA \\ ${ }^{2}$ Department of Mathematics, University of Michigan, Ann Arbor, MI 48109-1003, USA
}

In this paper we study the Hilbert space of analytic functions with finite Dirichlet integral in a connected open set $\Omega$ in the complex plane. We show that every such function can be represented as a quotient of two bounded analytic functions, each of which has a finite Dirichlet integral. This has several consequences for the structure of invariant subspaces of the algebra of multiplication operators on the Dirichlet space, in case $\Omega$ is simply connected. Namely, we show that every nontrivial invariant subspace contains a nontrivial bounded function, that each two nontrivial invariant subspaces have a nontrivial intersection (that is, the algebra is "cellular indecomposable"), and that each nontrivial invariant subspace has the "codimension one" property with respect to certain multiplication operators.

\section{Introduction}

Let $\Omega$ be a connected open set in the complex plane, and let $H^{\infty}(\Omega)$ denote the algebra of bounded analytic functions in $\Omega$, with the supremum norm. By the Bergman space of $\Omega$, denoted $B(\Omega)$ or $L_{a}^{2}(\Omega)$, we mean the set of analytic functions in $\Omega$ that are square integrable with respect to area measure. With the $L^{2}$ norm this is a Hilbert space.

The Dirichlet space, denoted $D(\Omega)$, is the set of (single-valued) analytic functions in $\Omega$ whose first derivative is in $B(\Omega)$; equivalently, these are the functions that map $\Omega$ onto a region of finite area (counting multiplicity). This space becomes a Hilbert space with the following norm. Select some point $z_{0}$ in $\Omega$ and let

$$
\|f\|_{D}^{2}=\left|f\left(z_{0}\right)\right|^{2}+\left\|f^{\prime}\right\|_{B}^{2} .
$$

If $d A$ denotes area measure, then

$$
\left\|f^{\prime}\right\|_{B=}^{2}=\int_{\Omega}\left|f^{\prime}\right|^{2} d A
$$

is called the Dirichlet integral of $f$ in $\Omega$. 
Finally, we introduce the space $M(D(\Omega))$ of multipliers on $D(\Omega)$, that is, the space of those functions $\varphi$ in $\Omega$ such that $\varphi D(\Omega) \subset D(\Omega)$. Using the closed graph theorem one shows that if $\varphi$ is a multiplier then multiplication by $\varphi$ is a bounded operator on $D(\Omega)$. With the operator norm $M(D(\Omega))$ is a Banach algebra. Since point evaluations are multiplicative linear functionals on this algebra, the multipliers are bounded functions (in a commutative Banach algebra all nonzero multiplicative linear functionals have norm one). Also, since the constant function 1 is in $D(\Omega)$ we see that the multipliers are contained in $D(\Omega)$. Thus

$$
M(D(\Omega)) \subset H^{\infty}(\Omega) \cap D(\Omega) .
$$

If $g$ is a conformal map (that is, an analytic homeomorphism) of $\Omega$ onto some domain $G$, then composition with $g$ induces an isometric isomorphism of the Banach algebra $H^{\infty}(G)$ onto $H^{\infty}(\Omega)$. Also, the space $D(G)$ is mapped onto $D(\Omega)$ and the Dirichlet integral is preserved. In addition, $M(D(G))$ is mapped onto $M(D(\Omega))$, preserving its action on the Dirichlet space. Finally, if $g$ maps the distinguished point $z_{0}$ of $\Omega$ to the distinguished point $w_{0}$ in $G$, then $g$ induces a unitary map between the Dirichlet spaces, and an isometric isomorphism between the multiplier spaces.

In the special case when $\Omega$ is the unit disk, $\mathbb{D}$, if $f$ has the power series $f(z)=\Sigma \hat{f}(n) z^{n}$, then a calculation shows that

$$
\left\|f^{\prime}\right\|_{B}^{2}=\int_{\mathbb{D}}\left|f^{\prime}\right|^{2} d A=\pi \sum_{1}^{\infty} n|\hat{f}(n)|^{2} .
$$

Thus if $z_{0}=0$ then $\|f\|_{D}^{2}=|f(0)|^{2}+\pi \Sigma n|\widehat{f}(n)|^{2}$.

The Dirichlet space in $\mathbb{D}$ lies in a family $\left\{D_{\alpha}\right\}_{\alpha \in \mathbb{R}}$ of Hilbert spaces; the norm is given in terms of the power series coefficients:

$$
\|f\|_{\alpha}^{2}=\Sigma(n+1)^{\alpha}|\widehat{f}(n)|^{2} .
$$

For $\alpha=1$ one has the Dirichlet space (with an equivalent norm). Two other special cases are $\alpha=0$ (the Hardy space $H^{2}$ ), and $\alpha=-1$ (the Bergman space $B$ ). A brief survey of the spaces $D_{\alpha}$ is given in [9]. We note that $D_{\alpha}$ is an algebra, contained in the disc algebra, when $\alpha>1$.

In his dissertation [10] Carleson considered spaces $T_{\alpha}$ related to the spaces $D_{\alpha}$, $0<\alpha \leqq 1$. The space $T_{0}$ is the Nevanlinna class of meromorphic functions of bounded characteristic, and the space $T_{1}$ consists of those meromorphic functions that map $\mathbb{D}$ onto a region of finite spherical area, counting multiplicity (that is, $\left(1+|f|^{2}\right)^{-1} f^{\prime}$ is in $\left.L^{2}(\mathbb{D})\right)$; the spaces $\left\{T_{\alpha}\right\}$ decrease with increasing $\alpha$. Also, $D_{\alpha}$ is contained within $T_{\alpha}$ (this can be proved using the formulae on page 19 of [10]).

The Nevanlinna class contains $H^{\infty}$, the space of bounded analytic functions in $\mathbb{D}$, and every Nevanlinna function is the quotient of two bounded functions. Carleson found a partial analogue of this for the spaces $T_{\alpha}$ : each function in $T_{\alpha}$ is a quotient of two bounded functions, each of which is in $T_{\beta}$ for all $\beta<\alpha$ (see [10], Theorem 3.6). He conjectured that one could not take $\beta=\alpha$ here, however, since there seemed to be an essential difference between bounded functions in $T_{\alpha}$ and general functions in $T_{\alpha}$ with regard to the exceptional set where the radial limit may fail to exist. Indeed, he shows (Theorem 3.3, p. 34; also p. 28) that this set has outer 
capacity zero of order $(1-\alpha)$ for general functions in $T_{\alpha}$, whereas, at least for inner functions in $T_{\alpha}$, it has Hausdorff measure 0 of order $1-\alpha$. (For $\alpha=1$ one has logarithmic capacity and logarithmic measure, see p. 14 of [10]).

We show in Theorem 1 below that for $\alpha=1$ one can take $\beta=1$, at least for functions in the Dirichlet space $D$. Thus for $\alpha=1$ the question is reduced to asking whether each function in $T_{1}$ is the quotient of two functions in $D$ ? Curiously this sort of result is also of interest in the theory of transitive operator algebras initiated by Arveson [3]; see the remarks at the end of this paper.

\section{Dirichlet Functions as Quotients of Bounded Functions}

We now show that every function in $D(\Omega)$ is a quotient of two bounded functions in $D(\Omega)$; this is also valid when $\Omega$ is a Riemann surface. The proof works for more general classes of functions (for the Dirichlet space it can be simplified somewhat); we require two lemmas.

Let $E$ be a set of analytic functions in a region $\Omega$. We say that the set $E$ is solid, if $h \in E, g$ holomorphic in $\Omega$, and $|g(z)| \leqq c|h(z)|$ in $\Omega$, imply that $g \in E$. For example, the space of $p^{\text {th }}$ power integrable analytic functions (with respect to area measure in $\Omega$ ) is solid. If $E$ is a set of analytic functions in $\Omega$, then by $E_{1}$ we denote the set of all those analytic functions in $\Omega$ for which $f^{\prime} \in E$. We note that $H^{\infty}(\Omega) \cap E_{1}$ is an algebra whenever $E$ is a solid linear space of analytic functions on $\Omega$.

Lemma 1. Let $\Omega$ be a connected open set in the complex plane, let $E$ be a solid set of analytic functions in $\Omega$, and let $f \in E_{1}$. If there is a function $F \in H^{\infty}(\Omega)$, not identically zero, and a positive constant $c$, such that, for all $z \in \Omega$,

a) $|f(z)|^{\delta}|F(z)| \leqq c \quad$ for some $\delta, 0<\delta \leqq 1$,

b) $\left|F^{\prime}(z)\right| \leqq c\left|f^{\prime}(z)\right|$,

then $f=\varphi / \psi$, where $\varphi, \psi \in H^{\infty}(\Omega) \cap E_{1}$.

Proof. By choosing the constant $c$ larger, if necessary, we may assume that $|F| \leqq c$ in $\Omega$. Select a positive integer $n$ such that $n \delta \geqq 1$, and let $\varphi=f F^{n+1}, \psi=F^{n+1}$. We first show that $\psi \in H^{\infty}(\Omega) \cap E_{1}$. Indeed, $\psi \in H^{\infty}(\Omega)$ since $|\psi| \leqq c^{n+1}$ in $\Omega$. Also,

$$
\left|\psi^{\prime}\right| \leqq(n+1)\left|F^{n}\right|\left|F^{\prime}\right| \leqq(n+1) c^{n+1}\left|f^{\prime}\right| .
$$

Thus $\psi^{\prime} \in E$, and so $\psi \in E_{1}$, as desired.

Next we show that $\left|f F^{n}\right| \leqq c^{n}$ in $\Omega$. Indeed, if $|f(z)| \leqq 1$, then $\left|f F^{n}\right| \leqq|F|^{n} \leqq c^{n}$. On the other hand, if $|f(z)|>1$, then

$$
\left|f(z) F^{n}(z)\right| \leqq\left(|f(z)|^{\delta}|F(z)|\right)^{n} \leqq c^{n} .
$$

We now show that $\varphi \in H^{\infty}(\Omega) \cap E_{1}$. Indeed, $|\varphi|=\left|f F^{n}\right||F| \leqq c^{n+1}$. Also,

$$
\begin{aligned}
\left|\varphi^{\prime}\right| & \leqq\left|f^{\prime} F^{n+1}\right|+(n+1)\left|f F^{n} F^{\prime}\right| \\
& \leqq c^{n+1}\left|f^{\prime}\right|+(n+1) c^{n+1}\left|f^{\prime}\right| \\
& \leqq(n+2) c^{n+1}\left|f^{\prime}\right| .
\end{aligned}
$$

Thus $\varphi \in E_{1}$ which completes the proof. 
Lemma 2. Let $\Omega$ be a connected open set in the complex plane, let $E$ be a solid set of analytic functions in $\Omega$, and let $f \in E_{1}$. If there is a compact set $K$ having positive two dimensional Lebesgue measure, such that $K$ and $f(\Omega)$ are disjoint, then $f=\varphi / \psi$, where $\varphi, \psi \in H^{\infty}(\Omega) \cap E_{1}$.

Proof. We may assume that the complement of $K$ is connected (otherwise we replace $K$ by a totally disconnected compact subset, still of positive measure; the complement of this new set will be connected). By a result of Nguyen Xuan Uy [18] (see Hruščëv [16] for another proof), there exists $g$, holomorphic and not constant in the complement of $K$ (with respect to the extended plane), such that both $g$ and $g^{\prime}$ are bounded there. By subtracting a constant we may assume that $g(\infty)=0$. Let $F(z)=g(f(z))$. To complete the proof we show that $F$ satisfies the conditions of Lemma 1.

First, $F$ is bounded since $g$ is bounded. Now let

$$
g(w)=a_{1} w^{-1}+a_{2} w^{-2}+\ldots
$$

be the power series expansion of $g$ about infinity. Then $w g(w) \rightarrow a_{1}$ as $|w| \rightarrow \infty$. It follows that $f(z) F(z) \rightarrow a_{1}$ as $|f(z)| \rightarrow \infty$, and thus hypothesis a) of Lemma 1 is satisfied, with $\delta=1$. Since $F^{\prime}=g^{\prime}(f) f^{\prime}$, and $g^{\prime}$ is bounded, we see that hypothesis b) is satisfied also.

Remark. Nguyen [18] produces a bounded holomorphic function $g$ that satisfies a Lipschitz condition of order one in the complement of $K$; the boundedness of $g^{\prime}$ follows from this. E.P. Dolženko [12] proved that if $K$ has 2-dimensional measure zero, if $U$ is an open set containing $K$, if $g$ is holomorphic in $U \backslash K$ and satisfies a Lipschitz condition of order one there, then $g$ extends to be holomorphic on $K$ (see also Garnett [14], Chap. 3, Theorem 2.3, p. 66). One says that $K$ is a removable set for the class Lip 1. Dolženko also showed that for functions of class $\operatorname{Lip} \alpha, 0<\alpha<1$, the necessary and sufficient condition for removability is that the set have Hausdorff measure zero of order $1+\alpha$.

Theorem 1. If $f \in D(\Omega)$, then $f=\varphi / \psi$, where $\varphi, \psi \in D(\Omega) \cap H^{\infty}(\Omega)$.

Proof. Since $f(\Omega)$ has finite area, counting multiplicity, the complement has infinite 2-dimensional measure, and the result follows from Lemma 2.

Remarks 1 . The theorem is valid also when $\Omega$ is a Riemann surface; the proof is the same, though some details have to be reinterpreted in the language of differential forms. (An analytic function of $\Omega$ is said to have a finite Dirichlet integral if the 2form $d f \wedge d \bar{f}$ is integrable.)

2. In case $\Omega$ is simply connected one can avoid Nguyen's theorem, and base the proof on Lemma 1. Indeed, for $f \in D(\Omega)$ one can choose a compact set $K$, of positive 2-dimensional measure, disjoint from $f(\Omega)$. One can define the square root so that $k(z, w)=(w-f(z))^{-1 / 2}$ is continuous in $w$, and analytic in $z$, for $w \in K, z \in \Omega$. Let $F(z)=\int k(z, w) d A(w)$. One shows that the hypotheses of Lemma 1 are satisfied, with $\delta=1 / 2$.

3. A different proof for the simply connected case can be given, using a formula of Carleson. Indeed, by conformal invariance it is sufficient to consider the unit 
disk, $\mathbb{D}$. If $f \in D(\mathbb{D})$, then $f$ has boundary values almost everywhere. Let $\psi_{f}\left(e^{i t}\right)=1$, whenever $\left|f\left(e^{i t}\right)\right| \leqq 1$, and $\psi_{f}=|f|^{-1}$ otherwise; let $\psi(\mathrm{z})$ be the outer function determined by $\psi_{f}$. Then $|\psi(z)| \leqq 1$ in $\mathbb{D}$. Using Carleson's formula for the Dirichlet integral, [11], it is shown in [9] (Lemma 7 (a), (c), (d), p. 284) that in this situation one has $\psi, \psi f \in D(\mathbb{D}) \cap H^{\infty}$. (The discussion in [9] is incomplete: when considering the contribution of the inner factor to the formula in [11] one needs to remark that $|\psi f| \leqq|f|$ a.e. on $\partial \mathbb{D}$.) An advantage of this proof is that the denominator, $\psi$, has no zeros in $\Omega$; it is not clear how to achieve this in the generality of Theorem 1 .

See the remarks at the end of the paper for further comments and questions related to Theorem 1 .

\section{Invariant Subspaces}

In this section we assume that $\Omega$ is simply connected and we consider the algebra $M(D(\Omega))$ of multipliers on $D(\Omega)$ (see the Introduction). By the conformal invariance of the Dirichlet space and of the space of multipliers, we may restrict attention to the unit disk $\mathbb{D}$. Throughout this section $D$ will denote the Dirichlet space in $\mathbb{D}$. Here we have the special operator $M_{z}$ of multiplication by $z$. It is known that every multiplier is the limit, in the strong operator topology, of a sequence of polynomials in this special operator (see [25], Theorem 12, p. 90). Warning: the operator $M_{z}$ does not always operate on $D(\Omega)$. It never does when $\Omega$ is unbounded, since multipliers are always bounded. But even for rectifiable Jordan domains $M_{z}$ may fail to operate (see Theorems 1 and 10 of [4]).

We recall the family $\left\{D_{\alpha}\right\}$ of Hilbert spaces in $\mathbb{D}$, defined in the Introduction. For all $\alpha \in \mathbb{R}$ the map $M_{z}, f \mapsto z f$ defines a bounded linear transformation on $D_{\alpha}$. We shall denote this operator by $M_{z}$ or by $\left(M_{z}, D_{\alpha}\right)$. A subspace $\mathscr{M}$ of $D_{\alpha}$ is called invariant, if $\left(M_{z}, D_{\alpha}\right)$ maps $\mathscr{M}$ into itself.

By subspace we always mean closed subspace.

If $f \in D$ then $[f]$ denotes the closure of the polynomial multiples of $f$, that is, the smallest invariant subspace containing $f$.

If $g$ is an analytic function on $\mathbb{D}$ then $g_{r}$ denotes the function defined by $g_{r}(z)=g(r z), 0 \leqq r<1$. Since $g_{r}$ is analytic on the closed disc $\mathbb{D}^{-}$one shows that $g_{r} f \in[f]$ for all $f$ in $D$.

Lemma 3. If $f \in D, \varphi \in D \cap H^{\infty}$, and $\varphi f \in D$, then $\varphi_{r} f \rightarrow \varphi f$ and $\varphi f \in[f]$.

Proof. As noted above, $\varphi_{r} f \in[f]$, and so it will be sufficient to show that $\varphi_{r} f \rightarrow \varphi f$. If $h \in D$ with $h(0)=0$, then it follows from the definition of the Dirichlet norm given in the Introduction that

$$
\|h\|_{D}^{2}=\pi \Sigma n|\hat{h}(n)|^{2}=\left\|h^{\prime}\right\|_{B}^{2},
$$

where $B$ denotes the Bergman space. Thus we have

$$
\begin{aligned}
\left\|\varphi_{r} f-\varphi f\right\|_{D} & \leqq\left\|\varphi_{r}\left(f-f_{r}\right)\right\|_{D}+\left\|(\varphi f)_{r}-\varphi f\right\|_{D} \\
& =\left\|\left\{\varphi_{r}\left(f-f_{r}\right)\right\}^{\prime}\right\|_{B}+\left\|(\varphi f)_{r}-\varphi f\right\|_{D} \\
& \leqq\left\|\left(\varphi_{r}\right)^{\prime}\left(f-f_{r}\right)\right\|_{B}+\left\|\varphi_{r}\right\|_{\infty}\left\|\left(f-f_{r}\right)^{\prime}\right\|_{B}+\left\|(\varphi f)_{r}-\varphi f\right\|_{D} .
\end{aligned}
$$


As $r \rightarrow 1$ the first term in the last expression approaches 0 by Lemma 3, page 278 of [9]. It is trivial that the second and third terms tend to zero, and so $\varphi_{r} f \rightarrow \varphi f$ as $r \rightarrow 1$.

Theorem 2. Let $\mathscr{M} \neq\{0\}, \mathcal{N} \neq\{0\}$ be invariant subspaces for $\left(M_{z}, D\right)$. Then

a) $\mathscr{M} \cap H^{* 0} \neq\{0\}$,

b) $\mathscr{M} \cap \mathscr{N} \neq\{0\}$,

c) $z \mathscr{M}$ is a closed subspace of $\mathscr{M}$ of codimension 1 .

Proof. a) Let $f \in \mathscr{M}, f \neq 0$. By Theorem 1 there are functions $\varphi, \psi \in H^{\infty} \cap D$ such that $f=\varphi / \psi$. By the Lemma, $\varphi=\psi f \in[f] \subset \mathscr{M}$, as required.

b) By a) there are bounded functions $\varphi \in \mathscr{M}$ and $\psi \in \mathscr{N}$. By the Lemma we have $\varphi \psi \in[\varphi] \cap[\psi] \subset \mathscr{M} \cap \mathscr{N}$, as required.

c) A calculation shows that $\|z f\|_{D} \geqq\|f\|_{D}$ for all $f \in D$; thus $z \mathscr{M}$ is a closed set. The codimension one property stated in c) follows from b) and a theorem of Bourdon [8].

\section{Remarks and Questions}

As operator is called cellular indecomposable if every two nontrivial invariant subspaces have a nontrivial intersection (see [19]). Thus Theorem $2 \mathrm{~b}$ ) states that $\left(M_{z}, D_{\alpha}\right)$ is cellular indecomposable when $\alpha=1$. What about other values of $\alpha$ ?

As mentioned earlier the spaces $D_{\alpha}$ are algebras for $\alpha>1$. Thus if $f, g \in D_{\alpha}$ then $f g \in[f] \cap[g]$ (see Proposition 7, p. 275 of $[9])$. Thus $\left(M_{z}, D_{\alpha}\right)$ is cellular indecomposable when $\alpha>1$.

Furthermore, $\left(M_{z}, H^{2}\right)$ is cellular-indecomposable for a similar reason. In fact, every invariant subspace contains an $H^{\infty}$ function, and $H^{\infty}$ coincides with the set of multipliers on $H^{2}$.

For $\alpha<0$ the operators $\left(M_{z}, D_{\alpha}\right)$ are not cellular indecomposable. This was first established by Horowitz [15]; it is also a consequence of the results in Chapter 10 of [6].

Conjecture 1. $\left(M_{2}, D_{\alpha}\right)$ is cellular indecomposable for $0<\alpha<1$.

We now make some comments about part c) of Theorem 2. One verifies that the operator $M_{z}-\lambda$ is bounded below on $D_{\alpha}$ for all $\lambda \in \mathbb{D}$ and all real $\alpha$. Hence if $\mathscr{M}$ is an invariant subspace for $\left(M_{z}, D_{\alpha}\right)$, then $\left(M_{z}-\lambda\right) \mid \mathscr{M}$ is bounded below and thus is a semi-Fredholm operator. Therefore the number

$$
\operatorname{dim}\left(\mathscr{M} \ominus\left(M_{z}-\lambda\right) \mathscr{M}\right)=\operatorname{dim} \operatorname{ker}\left(\left(M_{z}-\lambda\right) \mid \mathscr{M}\right)^{*}=-\operatorname{index}\left(\left(M_{z}-\lambda\right) \mid \mathscr{M}\right)
$$

does not depend on $\lambda \in \mathbb{D}$.

We say that an invariant subspace $\mathscr{M}$ of $\left(M_{z}, D_{\alpha}\right)$ has the codimension one property, if $\operatorname{dim} \mathscr{M} \ominus z M=1$ or if $\mathscr{M}=\{0\}$.

Thus Theorem $2 \mathrm{c}$ ) states that every invariant subspace of $\left(M_{z}, D\right)$ has the codimension one property. 
Invariant subspaces with the codimension one property are of a simpler structure than general invariant subspaces. In the few cases where a complete function theoretic description of all invariant subspaces is known $\left(H^{2}\right.$ : Beurling [7]; $D_{2}$ : Korenblum [17]) all invariant subspaces have the codimension one property. (See Šamoyan [23] for an extension of Korenblum's work to other algebras of analytic functions; see also Aleksandrov [2]). On the other hand, it follows from results of Apostol, Bercovici, Foias, and Pearcy (see Chap. 10 of [6]) that $\left(M_{z}, D_{\alpha}\right)$ has invariant subspaces which do not have the codimension one property, for each $\alpha<0$.

For more information on the significance of the codimension one property see $[22]$.

Conjecture 2. Every invariant subspace of $\left(M_{z}, D_{\alpha}\right)$ has the codimension one property, $0<\alpha<1$.

Invariant subspaces of $\left(M_{z}, D_{\alpha}\right)$ having the form $[f]$ are said to be cyclic (or singly generated). Since $[f]$ is the span of $\left\{f, z f, z^{2} f, \ldots\right\}$ it is easy to verify that $[f]$ has the codimension one property.

Conjecture 3. Every invariant subspace of $\left(M_{z}, D_{\alpha}\right)$ is singly generated, for $0<\alpha \leqq 1$.

The first and third conjectures each imply the second. Note that the third conjecture is open even for $\alpha=1$.

Next we say a few words about a possible extension of Theorem 1 suggested by the theory of operator algebras. An algebra $\mathscr{A}$ of operators (on a Banach space $E$ ) is said to be transitive if no proper subspace is invariant for all the operators in $\mathscr{A}$. The transitive algebra problem asks whether a transitive algebra must be dense (in the strong operator topology) in the algebra of all operators on $E$. An affirmative answer would imply that every operator on $E$ has a nontrivial invariant subspace (and much more). Some Banach spaces admit operators with no nontrivial invariant subspaces (see Beauzamy [5], Enflo [13], Read [20], [21]), thus the transitive algebra problem has a negative answer for such spaces. The problem is still open for reflexive Banach spaces.

One usually makes some additional assumption about the algebra $\mathscr{A}$. For example, Arveson [3] showed that if $\mathscr{A}$ contains the unilateral shift operator on Hilbert space, then $\mathscr{A}$ must be strongly dense. Ever since it has been an open problem whether his result remains true if $\mathscr{A}$ contains a weighted shift operator. Thus far this is only known for strictly cyclic shifts, which includes $\left(M_{z}, D_{\alpha}\right), \alpha>1$. In particular it is unknown for $\left(M_{z}, D_{\alpha}\right), 0<\alpha \leqq 1$, (and for $\alpha<0$ ). The unilateral shift may be identified with $M_{z}$ on $H^{2}$, and Arveson's proof used the fact that each function in $H^{2}$ is the quotient of two bounded functions, that is, of two multipliers on $H^{2}$. The proof would work for $\left(M_{z}, D_{\alpha}\right), 0<\alpha \leqq 1$, if the following conjecture were correct.

Conjecture 4. Each $f \in D_{\alpha}$ is the quotient of two multipliers on $D_{\alpha}$.

Recall that the multipliers on $D_{\alpha}$ are contained in $D_{\alpha} \cap H^{\infty}$. Thus this conjecture represents a generalization of Theorem 1 (for $\alpha=1$ ). 
The multipliers on $D_{\alpha}$ have been characterized by Stegenga [26]. For $0<\alpha<1$ even the direct analogue of Theorem 1 is open.

Conjecture 5. Each $f \in D_{\alpha}$ is the quotient of two bounded functions in $D_{\alpha}$.

Conjecture 6. Each invariant subspace of $\left(M_{z}, D_{\alpha}\right)$ contains a nontrivial multiplier $(0<\alpha \leqq 1)$.

This is correct for $\alpha>1$ and for $\alpha=0$. It is false for $\alpha<0$ (this follows from Theorem 6 of [24]; see also Horowitz [14]).

Conjecture 7. If $\Omega$ is a plane region (or Riemann surface) that has nonconstant analytic functions with finite Dirichlet integral, then it has nonconstant multipliers of the Dirichlet space.

It follows from Theorem 1 that $\Omega$ admits nonconstant $H^{\infty}$-functions with finite Dirichlet integral, but such functions are not necessarily multipliers. Ahlfors and Beurling [1] (Theorem 4) have characterized the plane domains $\Omega$ for which $D(\Omega)$ is nontrivial: there must be another domain conformally equivalent to $\Omega$, whose complement has positive two dimensional measure.

\section{Bibliography}

1. Ahlfors, L.V., Beurling, A.: Conformal invariants and function-theoretic null sets. Acta Math. 83, 101-129 (1950). MR 12, p. 171

2. Aleksandrov, A.B.: Invariant subspaces of shift operators. An axiomatic approach. Zap. Naučn. Sem. Leningrad Otdel. Math. Inst. Steklov (LOMI) 113, 7-26, 264 (1981) (Series title: Investigations on linear operators and the theory of functions, vol. XI.) Russian with English summary. MR $83 \mathrm{~g}: 47031$

3. Arveson, W.B.: A density theorem for operator algebras. Duke Math. J. 34, 635-647 (1967). MR 36 \#4345

4. Axler, S., Shields, A.L.: Univalent multipliers of the Dirichlet space. Mich. Math. J. 32, 65-80 (1985). MR 86c:30043

5. Beauzamy, B.: Un operateur sans sous-espace invariant: simplification de l'example de P. Enflo. Integral Equations Oper. Theory. 8, 314-384 (1985)

6. Bercovici, H., Foias, C., Pearcy, C.M.: Dual algebras with applications to invariant subspaces and dilation theory, CBMS Regional Conf. Ser. Math, vol. 56 (1985), Am. Math. Soc., Providence, Rhode Island

7. Beurling, A.: On two problems concerning linear transformations in Hilbert space. Acta Math. 81, 239-255 (1949) MR 10, p. 381

8. Bourdon, P.: Cellular-indecomposable shifts and Beurling's theorem. Mich. Math. J. 33, 187-193 (1986)

9. Brown, L., Shields, A.: Cyclic vectors in the Dirichlet space. Trans. Am. Math. Soc. 285, 269-304 (1984) MR 86d: 30079

10. Carleson, L:: On a class of meromorphic functions and its associated exceptional sets. Appelbergs Boktryckeri, Uppsala, 1950. MR 11, p. 427

11. Carleson, L.: A representation formula for the Dirichlet integral. Math. Z. 73, 190-196 (1960). MR 22 \#3803

12. Dolženko, E.P.: On the removal of singularities of analytic functions. Uspehi Mat. Nauk 18, 135-142 (1963); English translation: Am. Math. Soc. Transl., New Series 97, $33-41$ (1970). MR 27 \#5898

13. Enflo, P.: On the invariant subspace problem in Banach spaces. Acta Mathematica. To appear

14. Garnett, J.: Analytic capacity and measure. Lecture Notes Math. 297, Berlin-Heidelberg-New York: Springer 1972. MR 56\#12257

15. Horowitz, C.: Zeros of functions in Bergman spaces. Duke Math. J. 41, 693-710 (1974). MR 50 \#10215 
16. Hruščèv, S.V.: A simple proof of the theorem on removable singularities for analytic functions satisfying a Lipschitz condition. Akad. Nauk SSSR, Leningrad Otdel., Zapiski nauč. sem. LOMI 113, Issled. lin. op. teor. funk. XI, 199-203 (1981). (Russian, with English summary). MR 84d: 30082

17. Korenblum, B.: Invariant subspaces of the shift operator in a weighted Hilbert space. Mat. Sbor. 89(131), 110-137 (1972); English translation: Math USSR Sb. 18, 111-138 (1972). MR 47 \#5621

18. Uy, N.X.: Removable sets of analytic functions satisfying a Lipschitz condition. Arkiv Mat. 17, 19-27 (1979). MR 8li: 30076

19. Olin, R.F., Thomson, J.E.: Cellular-indecomposable subnormal operators. Integral Equations Oper. Theory. 7, 392-430 (1984). MR 85m: 47016

20. Read, C.J.: A solution to the invariant subspace problem on the space $l^{1}$. Bull. Lond. Math. Soc. 17, 305-317 (1985)

21. Read, C.J.: A short proof concerning the invariant subspace problem. J. Lond. Math. Soc. (2)34, 335-348 (1986)

22. Richter, S.: On invariant subspaces of multiplication operators on Banach spaces of analytic functions. Ph.D. dissertation, University of Michigan, 1986

23. Samoyan, F.A.: Closed ideals in algebras of analytic functions that are smooth on the boundary. Izv. Akad. Nauk Arm. SSR Ser., Mat. 16, 173-191 (1981). MR 83b: 46074

24. Shapiro, H.S., Shields, A.L.: On the zeros of functions with finite Dirichlet integral and some related function spaces. Math. Z. 80, $217-229$ (1962). MR 26 \#2617

25. Shields, A.L.: Weighted shift operators and analytic function theory. Topics in operator theory, 49-128. Math. Surveys 13, (1974) Am. Math. Soc., Providence, R.I. MR50 \#14341

26. Stegenga, D.A.: Multipliers of the Dirichlet space. Ill. J. Math. 24, 113-139 (1980). MR 81a: 30027

Received June 23, 1987 